\title{
QoS Routing for Data Gathering with RPL in WSNs
}

\author{
Miklós Molnár
}

\begin{abstract}
Generally, data collected by sensors must be sent to a sink node using multi-hop routing. Some applications can raise different requirements, constraints for the quality of data forwarding, for instance on end-to-end delay, jitter, packet loss, etc. Often, a set of constraints must be satisfied. Several proactive and reactive routing protocols have been proposed for WSNs. One of them is the standardized proactive RPL (Routing Protocol for Low-Power and Lossy Networks) designed essentially for many to one communication. For the data gathering a destination oriented directed acyclic graph (DODAG) is used which is mainly a tree directed to the sink. The computation of the tree-like DODAG is based on an Objective Function which can also be defined on QoS metrics but is usually based on one metric. This chapter deals with QoS routing in general and in WSNs using RPL in particular and proposes the analysis of the QoS constrained routes. We demonstrate that the set of QoS routes from sensors to the sink is not always a tree. It corresponds to a generalization of trees: to a hierarchy. The QoS aware hierarchies can be considered as special DODAGs. The routes are directed to the sink, and there is no useless cycle. The configuration of them and the data forwarding need some adjustments of the routing protocol which are also presented.
\end{abstract}

\section{Introduction, motivation}

The principal mission of Wireless Sensor Networks (WSNs) is measurement, monitoring and collection of data involved. In many applications data must be sent to a sink node, typically to a base station or to a border router. WSNs have many applications needing a defined Quality of Service (QoS). These applications are from environment, health and object monitoring and measurement to intrusion detection,

Miklós Molnár

LIRMM, University of Montpellier, CNRS, Montpellier, France e-mail: molnar@lirmm.fr 
multimedia applications in industrial control systems, real-time surveillance and alerts, etc.

From the point of view of network services, QoS characterizes the guaranties / requirements that the network should offer / satisfy to the end users of a service. QoS may be defined by a set of conditions to fulfill by a communication between a source and a destination (Crawley et al., 1998). Several elements are available to network operators to provide QoS in the network and in different OSI protocol layers. In our study, we consider that the majority of tools belongs to two main categories:

- tools using traffic engineering (TE) to manage the packet forwarding

- QoS aware routing to compute routes offering the asked guaranties.

TE proposes several tools to manage the traffic and help QoS. One can refer to classifications of flows, class based and/or priority based solutions, different policy based scheduling to handle queues, flow rate limitations to avoid congestions (e.g., flow control in TCP or traffic shaping). For reliability, acknowledgment based solutions are implemented to avoid packet losses in TCP and also in the CSMA/CA mechanism in some MAC layer protocols in wireless networks. Fault tolerance and reliability can be improved with multi-path routing and sending several copies of a message using independent routes.

The most known QoS aware solutions in the Internet are Intserv and Diffserv. Integrated Services (IntServ) is based on an individual reservation of resources for each flow using RSVP. This solution is not scalable, needs high resource consumption on the network nodes and can not be applied in WSNs. Differentiated Services or DiffServ is a more affordable solution to meet the QoS requirements in the Internet. The DiffServ approach operates on the principle of traffic classification, ensuring preferential treatment for higher-priority traffic classes (The Cisco Learning Network, 2017). The Software Defined Network concept permits to apply sophisticated mechanisms. A review of TE tools both in classic networks and Software Defined Networks can be found in (Abbasi et al., 2016).

Unfortunately, due to the limited capacities of the components, resourceintensive solutions of TE can not be applied to sensor networks.

Generally, WSNs are composed of autonomous, battery-powered, small and cheap sensors partially far from the base station. Sensors use short radio range to communicate. To collect data, multi-hop routing is needed. Moreover, some of the applications can raise different requirements for the quality of the measured data and also for the quality of the data forwarding mechanism. In our chapter we talk about the quality of the data forwarding, more especially of the routing in WSNs. The required QoS may include end-to-end delay, jitter, packet loss, or other critical parameters.

Frequently, the requirements are formulated as constraints and to forward data with respect to the QoS, not only one constraint but a set of constraints must be satisfied. Often, low power consumption is the primary objective of the routing decision, since the transmission of data from sensors to the sink is consumes important energy. 
For route computation, the used model is the topology graph with values assigned to the edges and nodes. Each constraint is based on a link or node metric and the set of constraints can be represented by vectors. The routing decision can be seen as a mono / multi objective multi-constrained optimization problem. In common cases, we suppose only one objective ( $c f$. Sect. 2). The computation of a simple path satisfying more than one constraint or an optimal (shortest) path under an additional constraint in the graph is NP-hard. In our scenario, a set of sensors should transmit data to the sink: the routing concerns a set of paths directed to the sink. We will use the term of incast communication to describe it. We consider the general case of the multi-constrained incast route computation when several QoS criteria are concerned in the optimization. The QoS incast route should contain a feasible path from each sensor to the sink node. An important property of the optimal (and also for some non optimal but feasible) solutions is that the set of feasible paths is not always a tree as it is expected, but may correspond to a generalization of trees: to a hierarchy (Molnár, 2011).

In this chapter we deal with QoS routing in general and QoS routing in WSNs using RPL in particular. The background of the multi-constrained path (and more generally route) computation is discussed shortly in Sect. 2. The optimal solution of the incast routing problem is also presented. QoS aware solutions in WSNs are over-viewed in Sect. 3. One of the standardized routing protocols proposed for lowpower and lossy networks is the protocol RPL (Routing Protocol for Low-Power and Lossy Networks) designed essentially for incast (many to one) communication (Gaddour and Koubâa, 2012). In Sect. 4 RPL is briefly presented and the QoS aware propositions using RPL are discussed in Sec. 5. For the data gathering a destination oriented directed acyclic graph (DODAG) is used which is mainly a tree directed from the sensors to the sink. The computation of the tree-like DODAG is based on an Objective Function which can be defined on some QoS metrics. Only a few Objective Functions are proposed and the existing functions are usually based on one metric (cf. examples in (Farooq et al., 2017)). In Sect. 5 we present how to satisfy several QoS constraints for data gathering using RPL. It is shown that the QoS aware hierarchies correspond to special DODAGs, the routes are directed to the sink, and there is no useless cycle. The configuration of these routes and the data forwarding need some adaptations of the routing protocol which are also presented.

\section{QoS constrained routing}

A lot of practical applications in recent networks require QoS and an important element to obtain the required quality is to make "good" routing decisions. In this section we resume the theoretical base of the QoS aware route computation. We are motivated by the computation of incast route. To introduce the multi-constrained incast routing problem, at first we propose a brief study of the multi-constrained path computation. Since there are important similitudes between broadcast and incast routes, we also present some significant results for QoS constrained broadcast / 
multicast routing, then we formulate the incast routing. In both cases (broadcast and incast), the optimal (and sometimes the existing feasible) solutions are not always trees. We present the route structure called hierarchy to precisely define the solution we are looking for.

\subsection{Graph model}

The appropriate model for route computations is based on the topology graph $G=$ $(V, E)$ of the network representing the node set $V$ and the links by the set $E$. The graph can be directed or not, depending on the real conditions of links. To simplify, we suppose an undirected graph with edge set $E$. Let us suppose that the state of the network is partially or entirely known to make routing decisions. Namely, the state of the links and nodes is described by appropriate parameters. Some of them correspond to QoS related metrics like the delay of transmission, the corresponding jitter, the probability of packet losses, etc. For the route computation, we suppose that:

- only the links have QoS parameters; if eventually there are parameters for the nodes, then each node can be replaced by an equivalent small complete graph in which the internal links are associated with the parameter set;

- the parameters are additive; multiplicative values can be transformed into additive ones using the logarithm function and bottleneck type parameters can be treated by link eliminations.

Frequently, the requirements are formulated as constraints on the end-to-end parameter values and not only one constraint but a set of constraints must be satisfied for the QoS. Often, low power consumption is the primary objective of the routing decision, since the transmission of data from sensors to the sink is very energy consuming. In these cases, the routing decision can be seen as a mono objective multi-constrained optimization problem in the valuated topology graph.

\subsection{Computation of constrained paths}

In the simplest case a QoS path should be found for a (source, destination) pair. The computation of a simple shortest path satisfying at least one additional constraint is an NP-hard problem. The determination of optimal paths is not suitable in large networks despite the fact that efficient models and methods have been proposed to solve it (Lozano and Medaglia, 2013). A significant part of QoS aware applications require multi-constrained routes. In a WSN, a set of sensors should transmit data to the sink: the routing concerns a set of paths directed to the sink satisfying a set of QoS constraints such as limited delay, jitter, bandwidth, loss rate etc. Evidently the computation of an optimal or even a feasible solution is a hard problem. 
In order to facilitate the discussion of the QoS aware incast route, in the next sub section, we propose a brief presentation of the optimal multicast route for two reasons. (1) The QoS aware multicast route computation has already been deeply analyzed, (2) Due to important similitudes, this analysis permit the definition of the optimal incast route for WSNs.

\subsection{Constrained multicast / broadcast}

It is well known that routes for multicast and broadcast are usually directed trees relaying the source to the destinations. Especially, these trees may be shortest path trees, minimum spanning trees (MSTs) which can be computed in polynomial time or approached minimum Steiner trees, knowing that the Steiner problem is NP-hard.

The QoS constraints strongly impact the multicast / broadcast routes. Since the computation of a single constrained shortest path is expensive, the shortest path tree under a QoS constraint is also difficult to compute. It is easy to see that the minimum cost multicast / broadcast route under a single QoS constraint is also a tree but this computation is NP-hard. To illustrate, the delay constrained multicast routing problem was first formulated in (Kompella et al., 1992). The diameter constrained Steiner tree problem is resumed in (Ding et al., 2010), where the diameter is the maximal distance of any node pair in the tree. The weight constrained minimum cost Steiner tree is analyzed in (Chen, Guangting and Burkard, Rainer E., 2003) where an additive arbitrary weight is limited on the arcs of the tree. Inversely, the minimum diameter cost constrained Steiner tree problem is presented in (Ding and Xue, 2012). The delay constrained minimum spanning tree (DCMST) problem where all the nodes in the network are concerned was introduced in (Salama et al., 1997). It is an interesting case for our study, because the data gathering in a WSN must cover the entire set of the nodes with the difference that in the data gathering the communications are oriented to the sink (root) node. Fig. 1 illustrates the DCMST for a broadcast in blue arrows and the delay constrained minimum cost incast tree in red. All the mentioned problems are NP-hard.

The computation is a more challenging and hard task when the communication should respect several constraints. Often, the proposed algorithms aim at the construction of a (minimum) spanning tree satisfying the set of QoS constraints. A recent work illustrates this construction of the spanning trees (Kumar and Singamsetty, 2018). Let us formulate the multi-constrained multicast / broadcast routing problem as follows.

\section{Definition 1 (The minimum cost multi-constrained multicast / broadcast prob- lem)}

Let $G=V, E)$ be a connected topology graph. A positive cost $c(e)$ and a vector $\vec{w}(e)=\left(w_{1}(e), w_{2}(e), \ldots, w_{k}(e)\right)^{T}$ of positive, additive weights are associated to each edge / arc $e \in E$. Let $s \in V$ be the source node and $D \subseteq V \backslash\{s\}$ the set of destinations. Let $\vec{L}$ be the vector of tolerated maximal QoS values on any path. 
Fig. 1 Illustration of a DCMST directed from a source to the other nodes (with blue arrows) and a delay constrained minimum cost incast tree directed from the nodes (sensors) to the sink (with red arrows) in the same non directed graph. The base of the two tree is the same DCMST, only the direction is different in the two cases

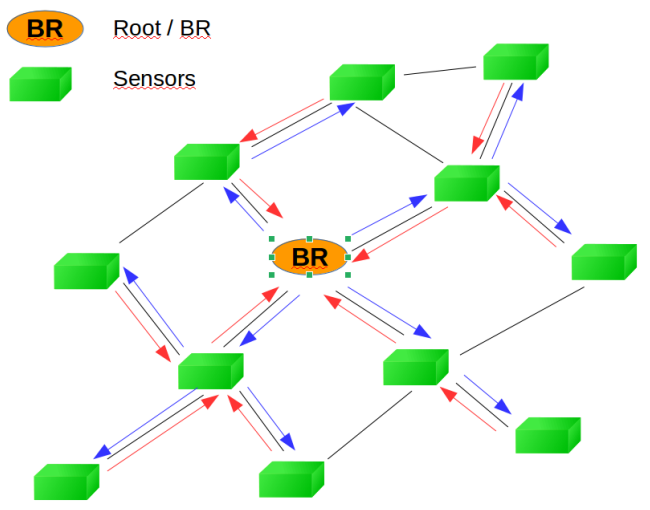

Consequently, a path from $s$ to $d \in D$ is a feasible QoS path, if its weight vector respects $\vec{w}\left(p_{s, d}\right) \stackrel{d}{\leq} \vec{L}$, where $\stackrel{d}{\leq}$ is the Pareto dominance. The minimum cost multiconstrained multicast / broadcast problem is to find a route offering a feasible path from the source to each destination minimizing the cost of the route.

Notice that the cost of the route should be defined. If the route is a sub-graph (a tree) and the packets take each edge of the sub-graph only once, then the cost of the route is the sum of the cost of edges composing it. We will demonstrate hereafter, that some optimal and also some feasible solutions are not trees nor sets of trees nor sets of QoS paths; they are not always sub-graphs and the communication cost should carefully be defined.

Deep analyses have been made for QoS constrained multicasting. Remember, multicast needs partial spanning (only the source and the destinations should be covered, the eventual intermediate relay node set is not fixed). The generalization for broadcasting is easy: the set of destinations corresponds to the set of nodes except the source. It was demonstrated, that a feasible partial spanning structure for multi-constrained QoS multicasting may be a sub-graph containing feasible paths from the source to the destinations (Kuipers and Mieghem, 2002). Unfortunately, the sub-graph is not sufficient to define the routes. It is true, a multi-constrained multicast route should provide a feasible path toward each destination but this route may not correspond to a sub-graph as it was demonstrated in (Molnár et al., 2012). The appropriate solution of the multi-constrained QoS routing problem is a graphrelated structure, which can precisely describe multicast / broadcast, and in our case, incast routes ( $c f$. in sub section 2.4 for incast communications). The solutions always correspond to hierarchies (Molnár, 2011). In some cases, the hierarchies can correspond to simpler routes: to paths and trees, which are particular cases of hierarchies. Similarly to multicasting, the spanning hierarchy concept enables to define the optimal solution. An illustration can be found in Fig. 2(d). Notice that the multi-constrained (partial) minimum spanning hierarchy problem is also NP-hard. Before going over the definition of hierarchy based QoS routes, let us define incast communications. 


\subsection{Multi-constrained incast route}

Many to one communication without QoS constraints (or with a single constraint) can use a tree for data forwarding to the root node. In our case the root of the tree is the sink in the WSN (e.g., a Border Router in RPL). The QoS constrained incast tree-based on one metric (e.g., on the latency) can be considered as an "inversed multicast / broadcast tree" as Fig. 1 suggests. The optimal incast route definition can be similar to the formulation in Definition 1, but there is an important difference.

The QoS constrained incast route, even if there is only one constraint, can be different from the inversed broadcast tree. The difference is due to the communication cost and the followed objectives.

- For cost optimized multicast / broadcast trees, the cost reflects the usage of the network resources: e.g., the sum of the edge costs in the tree. It is because an edge of the tree is used to transmit a message only once from the source to the destinations. This message is distributed, duplicated by the branching nodes in the tree.

- In incast routes (following the incast trees) the different messages traversing an edge near the sink and coming from different sensors use several times the edge: each message involves the cost represented by the edge (here we suppose that there is no aggregation of the messages in the network). The cost of the communication is the sum of the edges present in the set of paths.

- Several sets of paths for incast communication can implicate the same cost, and a different, routing related objective (e.g., the simplification of the routing tables) can also be interesting.

Here too, the presence of several QoS constraints modifies the problem and its solution. We propose the following formulation of the problem. In this formulation we talk about "routing aware" computation. We consider that an incast route is better for routing than another, if it contains less parent information (e.g., entries) in the set of nodes.

Definition 2 (The minimum cost, routing aware multi-constrained incast problem)

In the evaluated graph $G=V, E)$ the sink is the node $d \in V$ and the sources are the nodes in $S \subseteq V \backslash\{d\}$. The end-to-end QoS constraints are given by $\vec{L}$. The minimum cost routing aware multi-constrained incast problem is to find a route offering a feasible path from the sources to the unique destination minimizing the cost and also facilitating the routing:

$$
\min \left(u \cdot \sum_{p_{s, d} \in P_{Q o S}} C\left(p_{s, d}\right)+\sum_{v \in V \backslash\{d\}} N P(v)\right)
$$

where $P_{Q o S}$ is the set of QoS (feasible) paths in the solution, $C\left(p_{s, d}\right)$ is the cost of the path $p_{s, d}$, and $N P(v)$ is the number of parents in the node $v$. The weight $u$ is big enough to guarantee the priority of the cost component. 


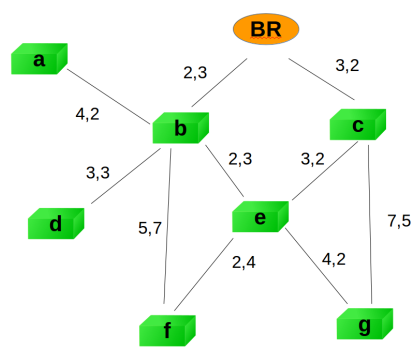

(a)

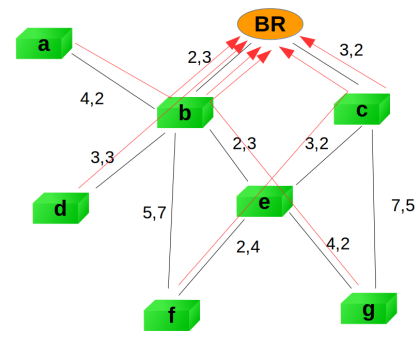

(b)

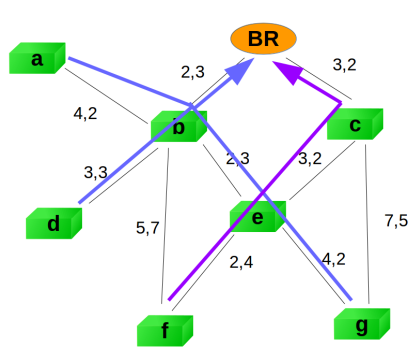

(c)

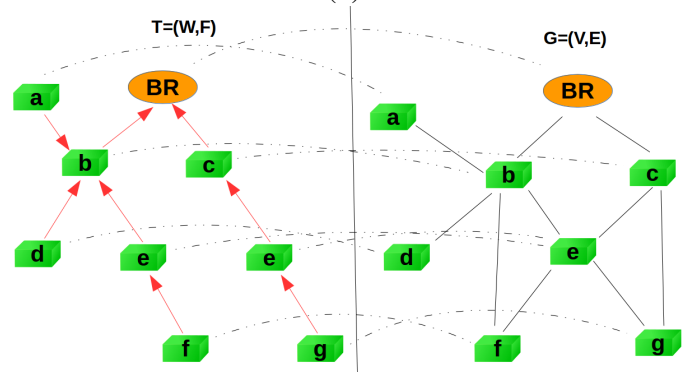

(d)

Fig. 2 (a) A WSN (b) The QoS paths to the BR (c) The route after the elimination of redundancies (d) The corresponding hierarchy

To find good solutions, it is indispensable to give the exact definition of the routes corresponding to the described optimization. This definition follows.

\subsection{Hierarchies to describe QoS routes}

At first, we demonstrate that trees can not always meet the end-to-end constraints for incast communications but in some cases, when there is no tree solution, hierarchies can.

Let a small network be considered as it is in Fig. 2(a). Two QoS related parameters are associated to the edges as it is indicated. The data gathering needs a QoS, the tolerated values are given by the vector $\vec{L}=[9,9]^{T}$. Each node should send messages to the BR respecting the constraints. Fig. 2(b) indicates the QoS paths from the nodes to the BR. There is only one feasible path from each node. In order to simplify the routing and share some edges, a reduced schema can be constructed as it is shown in Fig. 2(c). This schema is not a tree nor a set of paths. This structure is a destination oriented directed acyclic graph (DODAG). Fig. 2(d) illustrates how to obtain this route with the help of a hierarchy.

In a graph $G=(V, E)$ a hierarchy is defined as follows. 
Definition 3 (Hierarchy) Let $T=(W, F)$ be a tree (a connected graph without cycle). Let $x: W \rightarrow V$ be a homomorphism which associates a node $v \in V$ to each node $w \in W .{ }^{1}$ The association $(T, x, G)$ defines a hierarchy in $G$.

A hierarchy is not a sub-graph, it is a graph-related structure preserving some properties of trees but nodes and edges in the graph $G$ can be visited several times by a hierarchy. Trees are special hierarchies obtained by injective homomorphism. Thus, a tree is a hierarchy. Some properties in trees are true in hierarchies but all are not true. Conversely, all properties of hierarchies are true in trees. If the tree $T$ is directed in $(T, x, G)$, the defined hierarchy is also directed. For incast routing problems, hierarchies directed to the sink are needed.

In our first example in Fig. 2(d), Node $e$ is visited twice by the hierarchy. Since the different occurrences of the elements can play different roles, the distinction and the identification of the occurrences is substantial. For Node $e$ in the example: if a message is coming from Node $f$, it should be forwarded to $c$ and a message coming from $g$ should be forwarded to $b$ to satisfy the QoS constraints.

Generally, in routing problems, the graph $G$ is the topology of the network and the tree $T$ represents the routing information (the data forwarding). Our routing problem can be precisely formulated with the help of hierarchies.

The minimum cost, routing aware multi-constrained incast problem consists in finding the multi-constrained minimum directed spanning hierarchy containing at most one path $p\left(s_{j}, d\right)$ directed from each source $s_{j} \in S$ to the destination

$d$ respecting the constraints: $\vec{w}\left(p\left(s_{j}, d\right)\right) \stackrel{d}{\leq} \vec{L}$.

\section{QoS routing in WSNs}

The particularity of WSNs is that they are multi-hop, wireless networks without infrastructure to collect and send data to a sink or BR node using the network elements (sensors) as relays. The resources, i.e. the processing capacities, memories and battery powers are limited, reliability is low and the topology could change rapidly.

Route computation and selection impact strongly on the offered QoS. In the literature, several objectives are applied for QoS aware routing in WSNs. Some propositions go beyond the end-to-end quality measurement of routes. Important objectives as the energy utilization, the networks lifetime, the reliability, the stability of the paths are examined but in the recent chapter we focus our analysis on the usual set of end-to-end service oriented QoS parameters such as delay, jitter, packet loss, bandwidth, etc.

${ }^{1}$ A homomorphism is an association preserving the adjacencies of nodes; $w_{1} \in W$ and $w_{2} \in W$ can be adjacent (in T) iff the corresponding nodes $v_{1} \in V$ and $v_{2} \in V$ are also adjacent (in G) 
Routing protocols are presented and analyzed in several surveys classifying the most known propositions ( $c f$. an example in (Goyal and Tripathy, 2012)). An important aspect of the protocols is the timing of the route computation: proactive and reactive routing protocols are well known for WSNs. A concurrent reactive, on demand propositions and its analysis can be found in (Clausen et al., 2017). The protocol promises extensions to maintain collection trees and fast rerouting. The inconvenient of reactive protocol is the latency at least at the beginning of the communication. A large analysis of the WSN based applications and the proactive protocols can be found in (Mohamed et al., 2018). Hybrid solutions try to use the advantages of both strategies. An example is the zone-based ZRP which partitions the domain into a set of zones. Intra-zone routing uses a proactive protocol, and inter-zone routing is based on a reactive protocol diminishing the proactive image of the entire network. The QoS aspect of proactive, reactive and hybrid protocols for Mobile Ad Hoc Networks (MANETs) is compared in (Bhatia and Verma, 2015). Generally, reactive protocols outperform proactive ones in terms of throughput and packet delivery ratio whereas proactive protocols are better regarding end-to-end delay and traffic load.

Routing protocols in WSNs can also be classified as follows (there are only some aspects taken into consideration in this classification, it is not exhaustive). Destination-oriented routing is the usual solution in which the routing decision is based on a unique "address" and routing tables are often used to decide the next hop toward the destination. Routes can be constructed by a proactive procedure or on demand. Cost (or distance) - based solutions associate with each node a value proportional to the distance from the destination and the decision of the next hop tries to diminish this cost. For instance, the cost can be a rank as we will see in RPL (cf. Sect. 4). Geographic location (e.g., GPS) information can also be used to decide the next hop neighbor for data forwarding to the destination.

Often, cross-layer designs are proposed to achieve efficient solutions taking into account mechanisms in the neighbor layers (mainly in the MAC layer). We limit our study to the network layer.

Due to the very limited resources, the QoS aware routing in WSNs is a challenging task. The different applications require the satisfaction of different constraints. The energy minimization and the networks lifetime maximization are often important objectives. Some routing protocols for WSNs explicitly consider QoS requirements. Typically, the minimization of the energy usage is coupled with the respect of an end-to-end delay requirement. Sequential Assignment Routing (SAR) targets the QoS in the routing decisions (Sohrabi et al., 2000). It is a destination and multipath based protocol using trees rooted at the sink node. The data transmission is decided on the base of energy usage, an eventual QoS metric, and priority level of the packets. One QoS metric may be defined, for example, the delay, and SAR tries to minimize a weighted metric also calculated from the link cost and priority level of the packets. Moreover, this multi-path based protocol offers fault-tolerance and easy recovery, but it needs an important overhead to maintain routing tables. SPEED (Tian He et al., 2003) is a geographic routing protocol in WSNs providing soft end-to-end guarantees regarding the delay. Each node maintains information 
about its neighbors and uses geographic forwarding to find the paths. Soft real-time communication is achieved by maintaining a desired delivery speed. The end-to-end delay for the packets is estimated by dividing the distance to the sink by the speed of the forwarding. Real Time Power Aware Routing Protocol (RPAR) is an extension of SPEED to improve real time routing in WSNs. The principal element of this protocol is its capacity to adapt the transmission power and the routing decisions to the application delay requirements. For strong and short required delays it allocates more energy and capacity to respect the desired delay constraint (Chipara et al., 2006). In (Akkaya and Younis, 2003), an energy-aware QoS routing protocol has been proposed. The protocol finds a least-cost, delay-constrained path for real-time data. The link cost is a function based on the energy level of nodes, transmission energy, error rate and related parameters. The data forwarding supports two classes: best effort and real-time traffic at the same time, and a class-based queuing model is employed by adjusting the service rate for both classes.

Wireless Multimedia Sensor Networks pose additional challenges because the transmission of images and videos needs strong QoS. In (Alanazi and Elleithy, 2015) the authors state that the reliability and guarantee of end-to-end delay are critical while conserving energy. The propositions follow these objectives. The reader can find a large set of real-time QoS routing protocols classified into two categories, which are probabilistic and deterministic, including soft real time and hard real time QoS.

Only a few, really multi-constrained QoS routing algorithms for WSNs are known. In a few cases, algorithms to compute multi-constrained paths in wired network are proposed for WSNs. A little more often, one can find some works based on multiobjective QoS routing, when there are several objectives on the QoS metrics instead of multiple constraints. In (Alwan and Agarwal, 2013) a Multi-Objective QoS Routing protocol (MQoSR) is proposed. Multiple metrics are taken into account: energy, delay, reliability and hop count. The packets are forwarded to the next hop which minimizes a weighted sum representing the total cost. A multi-objective evolutionary algorithm uses ant colonies to solve the energy aware routing problem taking into account some aspects of QoS in (Su et al., 2013). Two performance metrics trying the maximization of the remaining lifetime of the network and also the minimization of the transmission delay are considered. In (Kulkarni et al., 2018) a heuristic routing algorithm called QoS assured Multi-objective Hybrid Routing Algorithm (Q-MOHRA) is proposed for heterogeneous WSN. This protocol takes into account link metrics as the needed energy, hop count, link quality indicator and path metrics as the jitter and uses a hierarchical clustering and a weighted sum of the used metrics to represent the goal.

In our opinion, the multi-objective formulation can not replace the multiconstrained one. The service related, end-to-end requirements are constraints and should (or could) not always be optimized. Moreover, there is no equivalence between the solutions: a solution of a multi-objective optimization obtained by a composite technique could not always meet all the constraints. Inversely, solutions respecting the constraints could be not optimal. The usual 
multi-objective optimization techniques propose trade offs: one non-dominated point on the Pareto front or a not optimal point behind this front is selected (and it depends on the applied technique: lexicographic, or weighted optimization, etc.)

In the following we focus on propositions handling multi-constrained cases. Moreover, since RPL is a standard for low-power and lossy networks (LLNs), we are interested with QoS routing using RPL. After a brief overview of the protocol, Sect. 5 presents some ideas for QoS aware RPL.

\section{RPL as a standard}

One of the standardized routing protocols for LLNs is the proactive, cost based RPL (Routing Protocol for Low-Power and Lossy Networks) proposed in RFC 6550 and it is designed essentially for incast (many to one) communication (Alexander et al., 2012). For data gathering, a destination oriented directed acyclic graph (DODAG) is used which is mainly a tree directed from the sensors to the sink. Nodes are organized into a "layered" hierarchical structure starting from a single root (the sink), finding direct children and children of children, etc. A node forwards the data to a parent (that has lower cost, i.e. lower rank) toward the sink.

A WSN using RPL can contain several RPL Instances for different applications, measurements. An RPL Instance may contain several DODAGs. A node can only belong to a single DODAG in an RPL Instance, but it can participate to multiple RPL Instances. In the protocol, Global RPL Instances are coordinated (containing one or more DODAG), but a Local RPL Instance corresponds always to a single DODAG and can be used for constructing DODAGs in support of a future on-demand routing solution (Alexander et al., 2012).

The construction of each tree-like DODAG is based on the attribution of "ranks" 2 descending from the root applying an Objective Function (OF) which can eventually be defined on QoS metrics. The OF gives the rules for the rank computation based on the routing metrics and it defines how to select a preferred parent for each node. Only a few OFs are proposed and the existing functions are usually based on one metric ( $c f$. examples in (Farooq et al., 2017)).

In RPL, the following ICMPv6 control messages are defined to construct and maintain the topology.

- DODAG Information Object (DIO). In order to determine the rank of nodes and select parents for data forwarding, the sink node starts the construction of its DODAG by broadcasting a DIO message to its neighbors. The rank of the sink is zero, and there is a version number for each DODAG. DIO includes information

\footnotetext{
2 the rank defines the position of the node in the DODAG with respect to the neighbors and the DODAG root ("hop" distance from the root)
} 
on the rank and also on the OF. This kind of messages are sent to the neighboring nodes descending from the sink in the topology. Based on the received messages and on the OF, nodes make the decision on the rank (which is incremented from the sink). A node selects a set of candidate parents (with ranks less to its rank) and a preferred parent used by default for data forwarding to the sink. Notice that DIOs may contain optional objects. For instance, an optional DAG Metric Container (DAGMC) contains eventual metrics and constraints. Metrics may be aggregated or recorded. An aggregated metric is cumulated on the path but for recorded metrics, each node adds a separated local value to the container. As the RFC indicates," RPL supports constraint-based routing where constraints may be applied to both links and nodes. If a link or a node does not satisfy a required constraint, it is "pruned" from the candidate neighbor set, thus leading to a constrained shortest path." The computed DODAG can be used for incast communications.

- Destination Advertisement Object (DAO).This message prepares the routes for down traffic and is used to propagate destination information upwards along the DODAG.

- DODAG Information Solicitation (DIS). When a new node tries to join an existing network, it sends a DIS message to its neighbors and requests graph information from its neighbors via DIO messages. After receiving the responses, the new node then selects its parent nodes.

Each node can have a set of candidate parents but only one preferred parent. If there is a failure, a local repair can change the preferred parent if there are other candidates. RPL includes also a global repair mechanism, which rebuilds the DODAG by incrementing its version.

For data gathering, a node sends data to the DODAG root passing by the configured consecutive preferred parents. Upward communications and node to node communications are also possible but they are out of scope of our chapter. For more details, we refer (Gaddour and Koubâa, 2012) and also (Kamgueu et al., 2018). The paper (Ghaleb et al., 2019) proposes a survey of some enhancements and limits of the protocol.

Arbitrary cost type metrics can be used in RPL for DODAG construction and the usage of constraints is possible but the effect of the constrained routing is limited. A constraint prevent the selection of paths passing by a node or link that do not meet the local requirement.

Basically, RPL uses special DODAGs, in which each node has one preferred parent. The data gathering follows a tree directed to the BR. For multiconstrained QoS routing more permissive solutions are needed. 


\section{QoS aware routing using RPL}

This section deals with QoS routing in WSNs using RPL. Our goal is to present solid proposals for cases when several constraints represent the QoS requirement. After reviewing previous papers illustrating the most significant propositions, we resume the theoretical bases of multi-constrained incast routing and some ideas how to compute and use the corresponding QoS routes in RPL.

\subsection{Propositions for QoS aware DODAGs}

Following the documentation of RPL, some simple OFs have been proposed and new OFs can be defined, such that the solutions should insure tree-based DODAGs.

Combinations of several routing metrics (into one composed metric) for QoS using RPL have been analyzed in (Karkazis et al., 2013). After a nice path algebra based presentation of routing problems, this study covers the usage of additive and lexicographic composition of metrics to compute QoS routes.

Remember, additive composite routing metrics on a path (link) $p$ can be defined as a weighted sum of $k$ primary metrics $w_{1}, w_{2}, \ldots, w_{k}$, like

$$
w(p)=a_{1} \cdot w_{1}(p)+a_{2} \cdot w_{2}(p)+\ldots+a_{k} \cdot w_{k}(p)
$$

where $a_{1}, a_{2}, \ldots a_{k}$ are positive constants.

For lexicographical composition, we simply indicate an alternative definition of the authors: "when multiple routing metrics are combined into a composite lexicographic routing metric, this dictates that the primary routing metrics are prioritized and when a path offers a better weight with respect to the first metric then it will be preferred regardless of the path weights of the rest metrics." This prioritization technique and the additive composition are often used for multi-objective optimizations. Trivially, the additive composition with adequate weights can be a possible implementation for the lexicographical composition.

Primary metrics like latency, remaining battery power, expected transmission count (ETX), ... are analyzed and used to make composite metrics to satisfy different requirements of the applications. The conditions for convergence to optimality and acyclic graph computation are also analyzed and proposed to use in RPL.

Often, the composite objectives are efficient to solve multi-objective optimization, but as we indicated in Sect. 3, these transformations can not always offer a solution for multi-constrained route computations. In the next section, we will show that a multi-constrained QoS incast route should permit the data forwarding to different parents. This possibility can not be insured by composite metrics.

Meta-heuristics may also be candidates to design QoS aware routing protocols. A protocol applying an ant colony optimization technique to compute routes in RPL is presented in (Mohamed and Mohamed, 2015). The proposed ant colony cooperation aims at finding a path at each node to the destination selecting a parent with a 
high probability of transition. For this, the probability of transition is expressed. The metrics used for the computation are the additive end-to-end delay and the bottleneck residual energy. Using the dynamic discovery of cooperating ants, the protocol determines energy efficient routes. The authors expect a better QoS using this solution. The respect of strict QoS constraints is not considered.

An original idea is to use a fuzzy inference system to choose the next hop of data forwarding. In (Gaddour et al., 2014) a novel objective function called OF-FL (OF based on fuzzy logic) combines a set of metrics for the routing decision of real-time, reliable communications with energy efficiency. Four metrics are considered: delay, ETX, hop count and remaining power of nodes as fuzzy variables. Another fuzzy inference system is applied in (Kamgueu et al., 2015) combining several metrics to decide the next hop (the preferred parent) using RPL. The proposed fuzzy inference system merges for instance expected transmission count, delay and remaining power of nodes into one unique value. Other criteria can also be used combining several metrics into one. The authors state that constraints and requirements are sometimes conflicting issues under a WSN context and it is sometimes difficult to decide the characteristic values of the fuzzy variables. Trade-offs must be found to balance between the QoS considerations. Fuzzy inference systems are simple, elegant tools for optimization even if several variables are considered and values are imprecise. They permit the combination of conflicting objectives and constraints and the complexity of the algorithms is low corresponding to the limitations of WSNs. However, the propositions can not cover cases, when different parents are needed in internal nodes for data transmissions coming from different sources ( $c f$. the sub section 5.2). Moreover, taking into account strict constraints with fuzzy logic based solution is not trivial.

Some works propose multi-path routing using RPL not only to improve QoS but also for a better fault-tolerance, enhancing reliability, and eventually decrease congestions. For instance (Iova et al., 2015) proposes a multi-path routing technique to allow RPL to forward data to multiple (preferred) parents. That is, multi-path routing in RPL involves multi-parent data forwarding. The main objective of this multi-parent routing is to improve the network lifetime by balancing the traffic load and avoiding the unbalanced depletion of the energy of nodes. For this, nodes should choose to send their traffic on the least energy limited path using an adequate path metric. Based on a given DODAG created in RPL, the data forwarding should balance the traffic toward all the existing parents. Another important statement by the authors is that frequent changes in the preferred parent may induce instability and higher energy consumption.

A combination of composite objectives and the fuzzy logic can be found in (Araújo et al., 2018). In this paper, four OFs are proposed for RPL which combine different metrics: ETX, number of hops and energy usage. The originality of the proposition is that OFs are dynamically selected based on contextual information. The authors say that fixed composition of metrics does not always correspond to the needs of an application. Information on the devices and on their environment can be used as contextual information for the route selection. The devices can select the preferred parent node (the applied OF) based on the contextual information. 
The paper proposes a route classifier based on fuzzy inference system to classify the possible routes and select the best one in each context. Notice, this solution can associate different next hop for data transmission, which is essential to satisfy multiple constraints being in contradiction. This proposition can be considered as a particular multi-path routing.

The most important part of the proposed solutions tries to find effective DODAGs by using composite metrics, meta heuristics and/or fuzzy systems for the data forwarding decisions. In these cases, the data forwarding uses a tree like graph with one preferred parent for each node. Trees can not always satisfy the multi-constrained $\mathrm{QoS}$ routing requirements. In some solutions, the set of parents can be used (multi-parent protocols) and a contextual decision making procedure has also been proposed. These propositions can help the adaptation of RPL to the more difficult multi-constrained routing cases, where a simple tree is not sufficient to meet the QoS requirement.

\subsection{Propositions for multi-constrained incast routing using RPL}

Section 2.4 defines the multi-constrained incast routing. As it is shown, finding a feasible solution is NP-complete and the computation of an optimal one is NP-hard. Eventual solutions are hierarchies directed to the BR. Remember, a hierarchy is defined by a triplet $(T, h, G)$. There are some trivial properties of the hierarchies solving the multi-constrained incast routing.

- Each directed path in the hierarchies is without loops, they form a DODAG. Possible crossings of directed paths in some nodes do not implicate loops.

- A node can have several parents in the graph $G$, but each node occurrence has only one parent in the tree $T$. Consequently, the decomposition of the hierarchy is always possible into a set of RPL compatible DODAGs ${ }^{3}$.

The adaptation of RPL Instances to meet the QoS constraints is required: a particular multi-parent solution is needed. Moreover, the computation of paths under several constraints is expensive and only non exact algorithms can be applied in WSNs.

\subsubsection{Path computation}

To compute multi-constrained QoS paths (which is NP-complete), and to control the satisfaction of the QoS requirements, a non-linear scalar length function has been proposed in (Kuipers and Mieghem, 2002). Let $\vec{L}$ be the $m$-dimensional vector of the

${ }^{3}$ We consider that a DODAG is RPL compatible, if each node has only one preferred parent 
QoS requirement and $\vec{w}(p(s, d))$ the weight vector of the path $p(s, d)$. Its non-linear length is:

$$
l(p(s, d))=\max _{i=1, \ldots, m} \frac{w_{i}(p(s, d))}{L_{i}}
$$

Trivially, if all the constraints are satisfied, then $l(p(s, d)) \leq 1$ and the path is feasible. To find a feasible solution, the shortest path from $s$ to $d$ using the nonlinear length can be computed. If this length is less than 1, the path is feasible (if the non-linear length of the shortest path is greater then 1, there is no feasible solution). To compute the shortest path, we follow the algorithm SAMCRA proposed in (Van Mieghem et al., 2001). It is a Dijkstra-like algorithm, but the greedy selection of shortest paths by the classical Dijkstra's algorithm can not be applied. To compare two paths arriving to a node, only the Pareto dominance can be used and all nondominated paths should be kept to continue the computation. The non-linear length based shortest path from $s$ to $d$ can be selected, when all non-dominated paths between the two nodes have been computed.

\subsubsection{Construction of multi-constrained DODAGs}

Unfortunately, the computation and the storage of all non-dominated paths in the nodes descending from the BR are not possible because of the limited capacities. In (Khallef et al., 2017b) a non-linear length based objective function has been presented to build a DODAG corresponding to a set of constraints. In the proposed construction, all of the non-dominated paths are not stored in the nodes. The DOI messages contain the cumulated weight vectors descending from the BR (for this, an optional DAG Metric Container in DIOs can be used). When a node received these messages from its potential parents, it computes the non-linear length of the received paths to the sink. The proposition supposes that the weights of the links are symmetric. Let $\vec{w}(p(u, d))$ be the weight vector from the node $u$ to the sink $d$. Let us suppose that this vector is sent to node $v$ via the link $e$ relying $u$ to $v$ having $\vec{w}(e)$ as weight vector. Trivially, the weight vector $\vec{w}\left(p^{u}(v, d)\right)$ associated with the path from the $v$ to $d$ passing by $u$ is:

$$
\vec{w}\left(p^{u}(v, d)\right)=\vec{w}(p(u, d))+\vec{w}(e)
$$

Based on the received values, $v$ can select all potential parent nodes corresponding to feasible paths (with non-linear length less then 1) and one preferred parent which corresponds to the minimal non-linear length. Only the weight vector passing by the preferred parent is then transmitted to the successors in the construction. Consequently, the algorithm can not enumerate all feasible paths and some solutions may be lost. However, a usual tree-based incast route is built by using the basic mechanism of RPL.

Following the example of the network shown in Fig. 2(a), the loss of feasible paths using the greedy algorithm is illustrated in Fig. 3(a). In this case, each node forwards by DIOs only one path (the path with minimal non-linear length) to the 
neighboring nodes. Node $e$ receives these single routes from $b$ and $c$. Since the two routes have the same non-linear length $(N L=6 / 9)$, it select one of them. Let us suppose that the route selected by $e$ to the sink is the route $(e, b, B R)$. This route can be a valid suffix for $g$ in the path $(g, e, b, B R)$. This path corresponds to a value $N L=8 / 9$. The same suffix is not suitable for Node $f$, since the path $(f, e, b, B R)$ has a non-linear length $N L=10 / 9$.

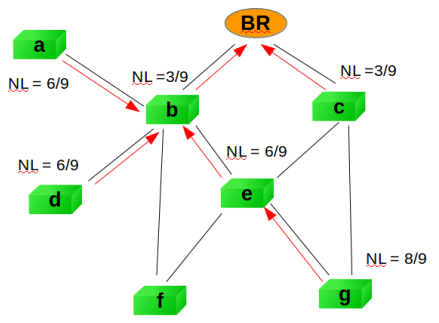

(a)

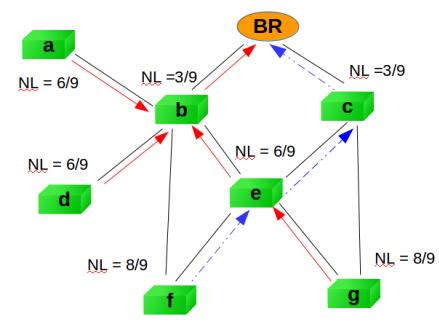

(b)

Fig. 3 (a) The result of the greedy NL based algorithm is an RPL DODAG (b) The decomposition of the exact solution into two mono parental RPL DODAGs. Notice that this solution can also be computed using the 2-limited heuristic

Since the computation of the multi-constrained incast hierarchy is NP-hard, trade-offs are needed between the quality of the routes and the computation time. In (Khallef et al., 2017a) the computation of the exact incast hierarchy is presented. To avoid the loss of theoretically reachable nodes, the exact solution does not limit the number of computed and stored feasible and non-dominated paths. To select preferred parents, the algorithm preserves all non reducible paths to the sink. In this way, a sensor can have a feasible path to the BR, if such a path exists. Since the exact algorithm can be very expensive, its interest is in the evaluation of inexact solutions. To find trade-offs between quality and computation time, parameterized polynomial time algorithms are proposed. The basic idea of the " $k$-limited algorithms" has been proposed for QoS path computation in the TAMCRA algorithm (Neve and Mieghem, 2000). It is a question of limiting the number of non-dominated feasible paths per nodes to $k$ paths, with a fixed positive integer $k$. Similarly to the exact computation, the obtained route does not always correspond to an RPL compatible DODAG but to a destination oriented directed hierarchy. In the $k$-limited version of the computation, a node receiving the non-dominated and feasible path propositions from the parents and computing its feasible paths can forward at most $k$ paths to its neighbors. (Notice that the exact solution corresponds to the case when $k$ is not limited.) As it is indicated in the paper, critical neighbors can find more probably a path corresponding to their needs, but it is always possible that there are unreached nodes because all the possible paths are not enumerated. 


\subsubsection{Evaluation of the propositions}

The proposed algorithms were evaluated in random network topologies. The random graphs were generated by the Waxman model. The presented results in (Khallef et al., 2017a) and presented also here are the mean values overs 100 instances. The 200 edges of the random graphs having 50 nodes were weighted by $m=2$ and in some other cases by $m=4$ integer QoS values. These values were also randomly generated from $\{1, \ldots, 10\}$. The most interesting results are expressed related to a derived metric: to the constraint looseness (CL). The CL is the average value of $L_{i} / \operatorname{maxv}_{i}, i=1, \ldots, m$, where $\operatorname{maxv}_{i}$ is the maximum possible value of metric of index i. A small value of CL indicates strict constraints and the maximal value of CL corresponds to cases when the constraint are not significant. Fig. 4(a) illustrates how many nodes can not be covered respecting different QoS requirement. When the constraints are strict, several nodes (probably the nodes far from the sink) are missing from the incast spanning hierarchies. Contrarily, with loose constraints the QoS routes can be "easily" built. Another lesson learned from this experiment is that the proportion of missing nodes decreases as the $k$ value of the limited heuristics increases. The average quality of the routes can be observed in Fig. 4(b). Remember that the non-linear length characterizes the most critical QoS value of the paths (it is less than 1 for feasible paths). The algorithms computing more alternative paths give better results. Moreover, in the case of strict QoS requirements, the non-linear length is greater than in the cases of loose constraints. Notice that the time of the computation is of the order of some minutes for the exact solutions and of a few milliseconds for the heuristics.

\subsubsection{Adaptation of RPL}

The presented solutions does not address an important element: how to use RPL to implement the computed route knowing that the latter may be a hierarchy? Remember, in the directed route, some graph nodes can have several parents as it is indicated in Fig. 3(b).

Here, the reader can find propositions for the creation and usage of the corresponding multi-parent RPL Instances.

One of the possible multi-parent data forwarding is the application of the contextual information ( $c f$. the idea in (Araújo et al., 2018)). In our case, the determinant context for the data forwarding decision is the origin of the data. This is a simple deterministic decision that does not need a fuzzy logic based classifier. In the example of Fig. 3(b), the only one node needing the contextual routing is Node $e$. The classification is simple: if a message arrives from Node $f$, then it should be forwarded to the parent $c$, otherwise the message should be transmitted to $b$.

A second possibility is the creation of several RPL Instances and DODAGs inside of the instances. The objective is the implementation of the directed hierarchy at the end of the route computation. This hierarchy can be decomposed into a set of RPL compatible DODAGs (remember, the difference is that in the loop less directed 


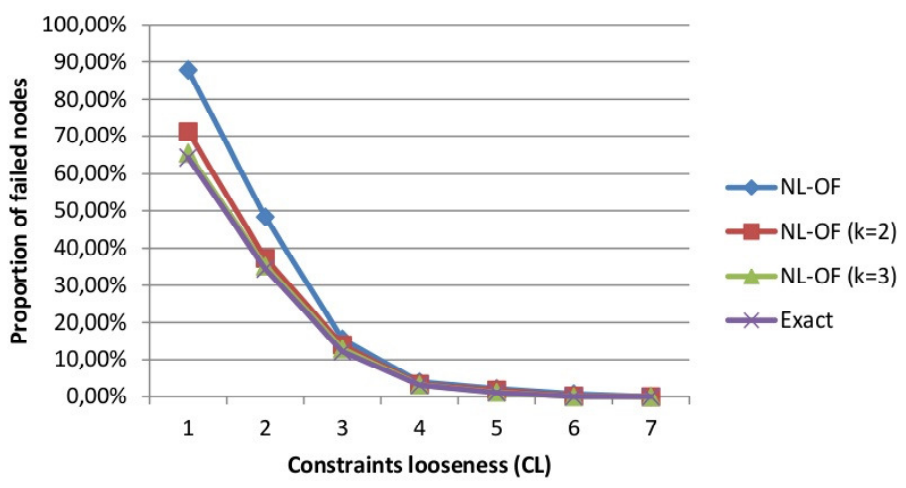

(a)

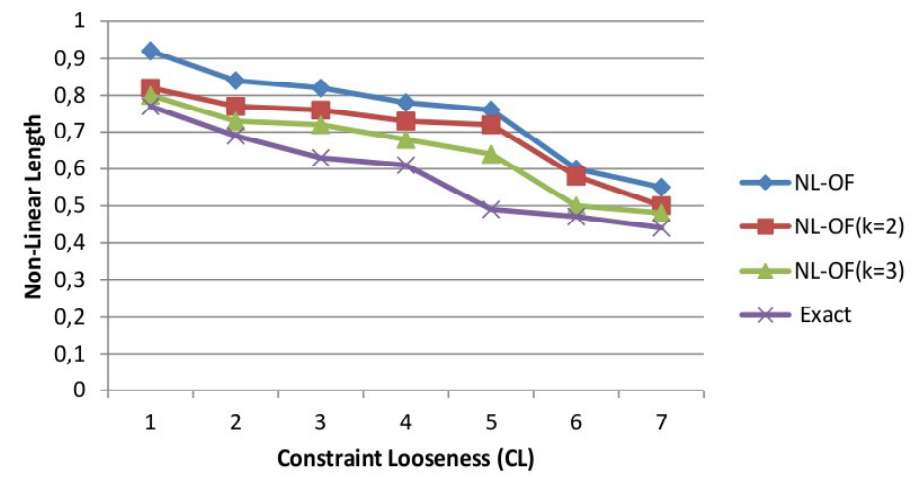

(b)

Fig. 4 (a) The ratio of missing (uncovered nodes) for different values of CL in the case of $m=4$ uncorrelated metrics (b) The non-linear length of the successfully computed paths. Results presented in (Khallef, 2017) and (Khallef et al., 2017a)

hierarchy, a node can belong to several paths to the root, but can not in an RPL compatible DODAG). To create the routing scheme of the example of Fig. 3(b), two RPL compatible mono parental DODAGs should be configured: a first indicated by red arrows and another indicated by blue arrows. In the following, a centralized route computation is briefly described.

1. Construct a Local RPL Instance beginning at the Route Computation Entity as root. To simplify, this entity may be the BR.

2. Propagate DIO messages and compute a $k$-limited set of QoS paths in the nodes, starting from the root (BR) of the future QoS constrained application. The selection of the preferred parents is temporary in this temporary DODAG.

3. Using the Local RPL Instance, send the set of $k$ feasible paths from each node to the Route Computation Entity.

4. Compute the global directed hierarchy as follows: 
a. The paths from leaves having only one QoS path to the BR should be selected and maintained in each traversed node

b. Leaves having a QoS path containing a shared common suffix with the existing hierarchy can be added to the hierarchy ${ }^{4}$. In the case of several candidate paths, select the path with minimal non-linear length. (Trivially, internal nodes of the added paths are added to the hierarchy.)

c. If there is no more leaf from which a path shares a common suffix with the hierarchy, then select a remained leaf and add to the hierarchy using its path with minimal non-linear length. (In this step, a new DODAG is initialized and multiple non compatible parents can be produced for some nodes.)

d. Re-iteration of the previous steps until all nodes are covered.

5. On the base of the computed hierarchy, create the needed RPL compatible DODAGs.

6. Send the RPL compatible DODAGs to the nodes to configure them.

Actually, using RPL mechanisms, RPL routers can not be configured explicitly. It can be candidate for future improvement of the protocol.

As the authors in (Iova et al., 2016) say, RPL needs a re-targeting. "For example, RPL could become a general standard framework, providing the glue for a suite of inter-related standards, each focused on specific communication technologies and/or application profiles." The QoS routing is a large domain to investigate in the protocols.

\section{Recent and future issues and challenges}

On one hand, in the domains of WSNs and IoT networks, with the development of applications increasingly demanding QoS, QoS-aware routing still requires considerable effort. For related basic research activities, one can mention some challenging needs.

As it is indicated in this chapter, the theoretical optimum for mono-objective multi-constrained incast routing corresponds to a directed hierarchy. The case of multi-objective routing requires further analysis. Moreover, finding efficient heuristics to build QoS-aware, hierarchy-based routes is an open domain. Notice that approximation guarantees for these routes are not known.

Eventual data aggregations in intermediate nodes can also modify the routing. The selection of efficient and appropriate aggregation techniques and the selection of the nodes that can execute them following appropriate routes can improve the performance of the data gathering. A large literature analysis of data aggregation in WSNs,

\footnotetext{
${ }^{4}$ Two directed paths share a suffix if the last sub-paths are the same in the two paths
} 
recent research issues and future research directions can be found in (Randhawa and Jain, 2017).

Network connectivity can change quickly and unpredictably. Note as cause the mobility and possible duty cycles of nodes. The use of opportunistic networks is a recent paradigm to deal with the problem. In opportunistic networks, the nodes transmit the messages when their connectivity is restored. Routing in this type of networks is challenging. A new routing strategy based on the node's activities in opportunistic networks is proposed in (Kumar et al., 2019). Compliance with QoS constraints in this type of network is a very difficult subject.

On the other hand, the evolution of applied network management techniques and of the sensor nodes and things, the use of increasingly intelligent solutions becomes feasible.

The monitoring of the network's state permits the detection of failures and disconnections, and the collect of QoS related parameters is essential for the routing decisions. The monitoring of the network's connectivity using RPL for applications serving critical missions is proposed in (Mostafa et al., 2018). The collect of QoS related values is a possible prolongation.

Software Defined Networking proposes an intelligent network management decoupling the control plane from the data plane, and thus using a central controller. A strong Border Router can be this controller. A comprehensive review of the Software Defined Networking in WSNs and its design requirements and challenges are analyzed in (Kobo et al., 2017).

One can also notice that with the evolution of systems used in WSNs, the application of TE based solutions (priority based routing, multi-path routing, ... becomes affordable.

\section{Conclusion}

QoS routing is an important and challenging topics in WSNs and IoT. Often, the QoS requirements correspond to a set of end-to-end constraints to respect. The standardized routing solution in LLNs is RPL. This protocol permits the definition of different Objective Functions and also constraints to build DODAGs mainly for incast (many to one) data gathering. The constraints relate edges and nodes but they are not end-to-end path related. Several QoS aware routing protocols are known and there are propositions for RPL. This chapter presents the problems of the propositions and also the real nature of the multi-constrained incast route corresponding to a graph related solution: to a hierarchy. Some elements of the computation of hierarchy based DODAGs are also presented. However there are open challenges to successfully deploy RPL in multi-constrained QoS requirements. Future investments are needed to find simple multi-parent solutions using RPL, to handle uncertainties by multi-path and opportunistic routing and to couple the routing to traffic engineering. 


\section{References}

Abbasi MR, Guleria A, Devi MS (2016) Traffic Engineering in Software Defined Networks: A Survey. Journal of Telecommunications and Information Technology 4:3 - 14

Akkaya K, Younis M (2003) An energy-aware QoS routing protocol for wireless sensor networks. In: 23rd International Conference on Distributed Computing Systems Workshops, 2003. Proceedings., pp 710-715, DOI 10.1109/ICDCSW.2003.1203636

Alanazi A, Elleithy K (2015) Real-Time QoS Routing Protocols in Wireless Multimedia Sensor Networks: Study and Analysis. Sensors 15(9):22209-22233, DOI 10.3390/s150922209

Alexander R, Brandt A, Vasseur J, Hui J, Pister K, Thubert P, Levis P, Struik R, Kelsey R, Winter T (2012) RPL: IPv6 Routing Protocol for Low-Power and Lossy Networks. RFC 6550, DOI 10.17487/RFC6550, URL https://rfc-editor.org/rfc/rfc6550.txt

Alwan H, Agarwal A (2013) Multi-objective QoS routing for wireless sensor networks. In: 2013 International Conference on Computing, Networking and Communications (ICNC), pp 10741079, DOI 10.1109/ICCNC.2013.6504241

Araújo HDS, Filho RH, Rodrigues JJPC, Rabelo RDAL, Sousa NDC, Filho JCCLS, Sobral JVV (2018) A Proposal for IoT Dynamic Routes Selection Based on Contextual Information. Sensors 18(2), DOI 10.3390/s18020353

Bhatia T, Verma A (2015) QoS Comparison of MANET Routing Protocols. International Journal of Computer Network and Information Security 7:64-73, DOI 10.5815/ijcnis.2015.09.08

Chen, Guangting, Burkard, Rainer E (2003) Constrained Steiner trees in Halin graphs. RAIRO-Oper Res 37(3):179-194, DOI 10.1051/ro:2003020

Chipara O, He Z, Xing G, Chen Q, Wang X, Lu C, Stankovic J, Abdelzaher T (2006) Realtime Power-Aware Routing in Sensor Networks. In: 200614th IEEE International Workshop on Quality of Service, pp 83-92, DOI 10.1109/IWQOS.2006.250454

Clausen T, Yi J, Herberg U (2017) Lightweight On-demand Ad hoc Distance-vector Routing-Next Generation (LOADng): Protocol, Extension, and Applicability. Elsevier Computer Networks 126:125-140, DOI 10.1016/j.comnet.2017.06.025

Crawley E, Nair R, Rajagopalan B, Sandick H (1998) A Framework for QoS-based Routing in the Internet. RFC 2386, Internet Engineering Task Force, URL http://www.rfceditor.org/rfc/rfc2386.txt

Ding W, Xue G (2012) On the Minimum Diameter Cost-Constrained Steiner Tree Problem. In: Lin G (ed) Combinatorial Optimization and Applications, Springer Berlin Heidelberg, Berlin, Heidelberg, pp 37-48

Ding W, Lin G, Xue G (2010) Diameter-Constrained Steiner Tree. In: Wu W, Daescu O (eds) Combinatorial Optimization and Applications, Springer Berlin Heidelberg, Berlin, Heidelberg, pp 243-253

Farooq MO, Sreenan CJ, Brown KN, Kunz T (2017) Design and analysis of RPL objective functions for multi-gateway ad-hoc low-power and lossy networks. Ad Hoc Networks 65:78 - 90, DOI https://doi.org/10.1016/j.adhoc.2017.08.002

Gaddour O, Koubâa A (2012) RPL in a nutshell: A survey. Computer Networks 56(14):3163 3178, DOI https://doi.org/10.1016/j.comnet.2012.06.016

Gaddour O, Koubâa A, Baccour N, Abid M (2014) OF-FL: QoS-aware fuzzy logic objective function for the RPL routing protocol. In: 2014 12th International Symposium on Modeling and Optimization in Mobile, Ad Hoc, and Wireless Networks (WiOpt), pp 365-372, DOI 10.1109/WIOPT.2014.6850321

Ghaleb B, Al-Dubai AY, Ekonomou E, Alsarhan A, Nasser Y, Mackenzie LM, Boukerche A (2019) A Survey of Limitations and Enhancements of the IPv6 Routing Protocol for Low-Power and Lossy Networks: A Focus on Core Operations. IEEE Communications Surveys Tutorials 21(2):1607-1635, DOI 10.1109/COMST.2018.2874356

Goyal D, Tripathy MR (2012) Routing Protocols in Wireless Sensor Networks: A Survey. In: 2012 Second International Conference on Advanced Computing Communication Technologies, pp 474-480, DOI 10.1109/ACCT.2012.98 
Iova O, Theoleyre F, Noel $\mathrm{T}$ (2015) Using multiparent routing in RPL to increase the stability and the lifetime of the network. Ad Hoc Networks 29:45 - 62, DOI https://doi.org/10.1016/j.adhoc.2015.01.020

Iova O, Picco P, Istomin T, Kiraly C (2016) RPL: The Routing Standard for the Internet of Things... Or Is It? IEEE Communications Magazine 54(12):16-22, DOI 10.1109/MCOM.2016.1600397CM

Kamgueu P, Nataf E, Ndie Djotio T (2015) On design and deployment of fuzzy-based metric for routing in low-power and lossy networks. In: 2015 IEEE 40th Local Computer Networks Conference Workshops (LCN Workshops), pp 789-795, DOI 10.1109/LCNW.2015.7365929

Kamgueu PO, Nataf E, Ndie TD (2018) Survey on RPL enhancements: a focus on topology, security and mobility. Computer Communications pp 1-17, DOI 10.1016/j.comcom.2018.02.011

Karkazis P, Trakadas P, Leligou HC, Sarakis L, Papaefstathiou I, Zahariadis T (2013) Evaluating routing metric composition approaches for QoS differentiation in low power and lossy networks. Wireless Networks 19(6):1269-1284, DOI 10.1007/s11276-012-0532-2

Khallef W (2017) Multi-Constrained Quality of Service Routing in Networks. Phd thesis, Université Montpellier, URL https://hal-lirmm.ccsd.cnrs.fr/tel-01887886

Khallef W, Molnár M, Bensliman A, Durand S (2017a) On the QoS routing with RPL. In: 2017 International Conference on Performance Evaluation and Modeling in Wired and Wireless Networks (PEMWN), pp 1-5, DOI 10.23919/PEMWN.2017.8308028

Khallef W, Molnár M, Benslimane A, Durand S (2017b) Multiple constrained QoS routing with RPL. In: 2017 IEEE International Conference on Communications (ICC), pp 1-6, DOI 10.1109/ICC.2017.7997081

Kobo H, Abu-Mahfouz A, Hancke G (2017) A survey on software-defined wireless sensor networks: Challenges and design requirements. IEEE Access 5:1872 - 1899, DOI 10.1109/ACCESS.2017.2666200

Kompella VP, Pasquale JC, Polyzos GC (1992) Multicasting for Multimedia Applications. In: Proceedings of the Eleventh Annual Joint Conference of the IEEE Computer and Communications Societies on One World Through Communications (Vol. 3), IEEE Computer Society Press, Los Alamitos, CA, USA, IEEE INFOCOM '92, pp 2078-2085

Kuipers FA, Mieghem PV (2002) MAMCRA: a constrained-based multicast routing algorithm. Computer Communications 25(8):802-811, DOI http://dx.doi.org/10.1016/S01403664(01)00402-9

Kulkarni N, Prasad NR, Prasad R (2018) Q-MOHRA: QoS Assured Multi-objective Hybrid Routing Algorithm for Heterogeneous WSN. Wireless Personal Communications 100(2):255-266, DOI 10.1007/s11277-017-5064-8

Kumar P, Chauhan N, Chand N (2019) Node activity based routing in opportunistic networks. In: Singh PK, Paprzycki M, Bhargava B, Chhabra JK, Kaushal NC, Kumar Y (eds) Futuristic Trends in Network and Communication Technologies, Springer Singapore, Singapore, pp 265-277

Kumar TJ, Singamsetty P (2018) An exact algorithm for multi-constrained minimum spanning tree problem. International Journal of Mathematics in Operational Research 12(3):317-330, DOI 10.1504/IJMOR.2018.10010953

Lozano L, Medaglia AL (2013) On an exact method for the constrained shortest path problem. Computers \& Operations Research 40(1):378 - 384, DOI https://doi.org/10.1016/j.cor.2012.07.008

Mohamed B, Mohamed F (2015) QoS Routing RPL for Low Power and Lossy Networks. Int J Distrib Sen Netw 2015:1-10, DOI 10.1155/2015/971545

Mohamed RE, Saleh AI, Abdelrazzak M, Samra AS (2018) Survey on wireless sensor network applications and energy efficient routing protocols. Wireless Personal Communications 101(2):10191055, DOI 10.1007/s11277-018-5747-9

Molnár M (2011) Hierarchies to Solve Constrained Connected Spanning Problems. Tech. Rep. RR-11029, LIRMM, URL https://hal-lirmm.ccsd.cnrs.fr/lirmm-00619806

Molnár M, Bellabas A, Lahoud S (2012) The cost optimal solution of the multiconstrained multicast routing problem. Computer Networks 56(13):3136 - 3149, DOI https://doi.org/10.1016/j.comnet.2012.04.020, Challenges in High-Performance Switching and Routing in the Future Internet 
Mostafa B, Benslimane A, Saleh M, Kassem S, Molnár M (2018) An Energy-Efficient Multiobjective Scheduling Model for Monitoring in Internet of Things. IEEE Internet of Things Journal 5(3):1727-1738, DOI 10.1109/JIOT.2018.2792326

Neve HD, Mieghem PV (2000) TAMCRA: a tunable accuracy multiple constraints routing algorithm. Computer Communications 23(7):667 - 679, DOI https://doi.org/10.1016/S01403664(99)00225-X

Randhawa S, Jain S (2017) Data aggregation in wireless sensor networks: Previous research, current status and future directions. Wireless Personal Communications 97(3):3355-3425, DOI $10.1007 / \mathrm{s} 11277-017-4674-5$

Salama HF, Reeves DS, Viniotis Y (1997) The delay-constrained minimum spanning tree problem. In: Proceedings Second IEEE Symposium on Computer and Communications, pp 699-703, DOI 10.1109/ISCC.1997.616089

Sohrabi K, Gao J, Ailawadhi V, Pottie GJ (2000) Protocols for self-organization of a wireless sensor network. IEEE Personal Communications 7(5):16-27, DOI 10.1109/98.878532

Su S, Yu H, Wu Z (2013) An Efficient Multi-objective Evolutionary Algorithm for Energyaware QoS Routing in Wireless Sensor Network. Int J Sen Netw 13(4):208-218, DOI 10.1504/IJSNET.2013.055583

The Cisco Learning Network (2017) QoS architecture models: IntServ vs DiffServ. https://learningnetwork.cisco.com/message/650042\#650042, online; accessed 22 June 2019

Tian He, Stankovic JA, Chenyang Lu, Abdelzaher T (2003) SPEED: a stateless protocol for real-time communication in sensor networks. In: 23rd International Conference on Distributed Computing Systems, 2003. Proceedings., pp 46-55, DOI 10.1109/ICDCS.2003.1203451

Van Mieghem P, De Neve H, Kuipers F (2001) Hop-by-hop Quality of Service Routing. Computer Networks 37(3-4):407-423 